\title{
Meriç Delta Balıklarında Toksik Metal Birikimlerinin Değerlendirmesi: Muhtemel İnsan Sağlığı Riskleri
}

\author{
Cem TOKATLI ${ }^{*}$, Fikret USTAOĞLU² \\ ${ }^{1}$ Laboratuvar Teknolojisi Proğramı, Trakya Universitesi, İpsala, Edirne, Türkiye \\ ${ }^{2}$ Biyoloji Bölümü, Giresun Universitesi, Giresun, Türkiye \\ *Sorumlu Yazar: tokatlicem@gmail.com
}

Araştırma Makalesi

Geliş 14 Temmuz 2020; Kabul 03 Kasım 2020; Basım 01 Mart 2021.

\begin{abstract}
Alıntılama: Jorfipour, M., Keivany Y., Paykan-Heyrati, F., \& Ghafouri, Z. (2021). Meriç delta balıklarında toksik metal birikimlerinin değerlendirmesi: Muhtemel insan sağlığ
\end{abstract} https://doi.org/10.22392/actaquatr.769656

\section{Özet}

Meriç Deltası Türkiye'nin Trakya bölgesinde yer alan dünya çapında öneme sahip sulak alanlardan biridir. Delta içinde yer alan Gala ve Sığırcı gölleri, biyo-çeşitlilik bakımından Türkiye'nin önemli göllerindendir. Bu çalışmada Sığırcı ve Gala göllerinden elde edilen altı balık türünün (Carassius gibelio, Carassius carassius, Scardinius erythrophthalmus, Cyprinus carpio, Sander lucioperca ve Perca fluviatilis) kaslarındaki toksik ve esansiyel element ( $\mathrm{Li}, \mathrm{B}, \mathrm{Al}, \mathrm{V}, \mathrm{Cr}, \mathrm{Mn}, \mathrm{Fe}, \mathrm{Co}, \mathrm{Ni}, \mathrm{Cu}$, $\mathrm{Zn}, \mathrm{As}, \mathrm{Se}, \mathrm{Sr}, \mathrm{Ag}, \mathrm{Cd}, \mathrm{Sb}, \mathrm{Ba}, \mathrm{Tl}$, ve $\mathrm{Pb}$ ) konsantrasyonları tespit edilerek, bu elementlerin tüketimi ile ilişkili potansiyel insan sağlığı riskleri, yetişkinlerde "tahmini günlük tüketim (EDI)" dikkate alınarak değerlendirildi. Ayrıca, genel popülasyon için balık tüketim sıklığı dikkate alınarak, "hedef tehlike katsayısı (THQ)", "tehlike indeksi (HI)" ve "kanserojen riski (CR)" değerleri belirlendi. Bu sonuçlara göre, tüm balık türlerin yenilebilir kaslarında $\mathrm{Li}, \mathrm{B}, \mathrm{Cr}, \mathrm{Co}, \mathrm{Se}, \mathrm{Tl}, \mathrm{ve} \mathrm{Pb}$ için EDI değerleri genel olarak izin verilen günlük doz limitini aşmıştır. Ancak 1'in altında tespit edilen THQ ve HI değerleri kanserojen sağlık riskinin olmadığını göstermiştir. Ayrıca, As, Cr, Ni ve Pb için CR değerleri kabul edilebilir sınırlardadır. Sonuçlar, Sığırcı ve Gala göllerindeki balıkların kaslarındaki toksik element konsantrasyonlarının tüketiciler için önemli bir sağlık riski oluşturmadığını ortaya koymuştur.

Anahtar Kelimeler: Gala ve Sığırcı Gölleri, Balıklar, Toksik Metaller, Sağlık Riski Değerlendirmesi

Evaluation of Toxic Metal Accumulations in Meriç Delta Fish: Possible Risks to Human Health

\section{Abstract}

Meriç Delta is in the Thrace region of Turkey and it is one of the wetlands of worldwide importance. Siğırcı and Gala lakes are in the delta and they are two of Turkey's most important wetland in terms of biodiversity. In this study, toxic and essential element concentrations(Li, B, Al, V, Cr, Mn, Fe, Co, Ni, Cu, Zn, As, Se, Sr, Ag, Cd, Sb, Ba, Tl, and Pb) in the muscles of six fish species (Carassius gibelio, Carassius carassius, Scardinius erythrophthalmus, Cyprinus carpio, Sander lucioperca, and Perca fluviatilis) obtained from Sı ğırcı and Gala lakes were determined and potential human health risks associated with the consumption of these elements were evaluated by calculating "estimated daily intake (EDI) in adults. In addition, considering the frequency of fish consumption for the general population, "target hazard quotient (THQ)", "hazard index (HI)" and "carcinogen risk (CR)" values were determined. According to these results, EDI values for $\mathrm{Li}, \mathrm{B}, \mathrm{Cr}, \mathrm{Co}, \mathrm{Se}$, $\mathrm{Tl}$, and $\mathrm{Pb}$ in all edible muscles of all fish species generally exceeded the allowable daily dose limit. However, THQ and HI values below 1 showed that there was no carcinogenic health risk. Also, $\mathrm{CR}$ values for $\mathrm{As}, \mathrm{Cr}, \mathrm{Ni}$ and $\mathrm{Pb}$ are within acceptable limits. The results revealed that the toxic element concentrations in the muscles of the fish in Siğırci and Gala lakes do not pose a significant health risk for consumers.

Keywords: Gala and Cattle Lakes, Fish, Toxic Metals, Health Risk Assessment

\section{GíRiș}

Son yıllarda, hızlı endüstrileşme, ekonomilerin yüksek kalkınma hızı, kentleşme, nüfus artışı ve tarımsal faaliyetler nedeniyle oluşan çevre kirliliği ve bununla ilişkili riskler, ciddi bir sorun haline gelmiştir (Çiçek vd., 2013; Köse vd., 2018; Ustaoğlu ve Islam, 2020). Ekosistemler, birçok kirleticinin baskısına maruz kalmıştır. Özellikle eser elementler (EE), toksisite, biyoakümülasyon ve biyomagnifikasyon özelliklerinden dolayı su ekosistemlerini (su, sediment, biyota) en çok kirleten kontaminantlar olarak öne çıkmıştır (Türkmen vd., 2008; 2012; Paramasivam vd., 2015; Ustaoğlu vd., 
2020a). EE; esansiyel ( $\mathrm{Zn}, \mathrm{Cu}, \mathrm{Fe}, \mathrm{Co} \mathrm{vb}$ ) ve esansiyel olmayan ( $\mathrm{Pb}, \mathrm{Cd}, \mathrm{As}, \mathrm{Hg}$ vb.) olarak sınıflandırılır. Her iki TE grubu da yüksek konsantrasyonlarda toksisiteye sebep olabilir. Sucul ortamlardaki EE'ler hem antropojenik faaliyetlerden (Örneğin sanayi ve endüstriyel atık suları, madencilik ve arıtma, tarımsal drenaj, evsel atıklar ve atmosferik birikim) hem de doğal kaynaklardan (genellikle toprak ve kayaların ayrışması, volkanik patlamalar, orman yangınları erozyon) kaynaklanır (Sundaray vd., 2011; Tokatl1, 2019; Ustaoğlu vd., 2020b).

İnsanların, EE'lere maruz kalmasının başlıca yollarında biriside gıda tüketimi ile olmaktadır. EE'ler, ortamda düşük konsantrasyonlarda olsalar bile, besin zincirindeki biyolojik birikim nedeniyle insanlar için sağlık riski oluşturabilir. Bu yüzden diyet yoluyla EE alınması risk değerlendirmesinde önemli bir konudur (Türkmen vd., 2010; Ahmed vd., 2015). Örneğin balıklar içerdikleri değerli proteinler, mikro elementler, vitaminler, omega-3 yağ asitleri kolayca sindirilen ve emilen doymamış yağ asitleri bakımından zengindir. Bu nedenle dünya çapında insan diyetinin çok önemli bir kısmını oluşturlar (Li vd., 2020). Ayrıca su ortamlarının önemli bir biyoindikatörü olarakta kabul edilirler. Ancak balıkların yaşadıkları ortamdan kolayca etkilendiği, çevrelerindeki su, sediment ve besinlerdeki ağır metal gibi zararlı kontaminantları biriktirdiği de bilinen bir gerçektir (Islam vd., 2015; Yin vd., 2020). Bu sebeple yüksek besin değerine rağmen, kontamine olmuş balıkların tüketimi dünya çapında ciddi bir halk sağlığı tehlikesi oluşturma potansiyeline sahiptir (Alamdar et al., 2017). Son y1llarda EE tarafından kontamine olmuş balıkların tüketimi ve risk değerlendirmesi ile ilgili pek çok araştırma yürütülmüsstür (Fang vd., 2017; Feng vd., 2020; Kosker, 2020; Maurya vd., 2019; Miao vd., 2020; Varol vd., 2020; Varol ve Sünbül, 2020, 2018, 2018; Yin vd., 2020; Zerizghi vd., 2020).

Göller su ürünlerinin ana kaynaklarından biri olmakla birlikte, çevresindeki insani aktivitelere karşıda bir o kadar hassastır. Göllerin hızlı endüstriyel ve tarımsal büyüme ile ağır metaller tarafından kirlendiği ve insan sağlığı için potansiyel bir tehdit oluşturduğu bildirilmiştir (Ali vd., 2019).

Meriç Deltası, Türkiye'nin Trakya Bölgesi'ndeki Meriç Nehri'nin ağzında yaklaşık 45.000 ha alanı içermektedir. Özellikle kuş varlığı açısından Türkiye'nin en zengin su yaşam alanlarından biridir. Uluslararası kriterlere göre "A Sınıfı Sulak Alan” olarak sınıflandırılmıştır (aynı anda 25.000'den fazla su kuşunu barındırabileceği anlamına gelir). Uluslararası öneme sahip bu ekosistem, tarımsal, evsel ve endüstriyel uygulamalar yoluyla yoğun bir kirliliğe maruz kalmaktadır. Meriç Nehri çevresinde yapılan çeltik tarımı ve Ergene Nehri Havzası çevresinde gerçekleştirilen endüstriyel faaliyetler, sistemi etkileyen ana kirlilik faktörleri olarak kabul edilmektedir. Türkiye'nin toplam pirinç üretiminin yaklaşık\% 25'i bu havzadan sağlanmaktadır. Ergene Nehri de Türkiye'nin en kirli nehir ekosistemlerinden biri olarak biliniyor. 2005 yılında Gala Gölü "Milli Park" olarak ilan edilmiş ve Avrupa ile Afrika arasında göç eden birçok kuş türü için konaklama yeri sağlamaktadır. Sığırcı Gölü de yerel balıkçılık için özel bir öneme sahiptir ve Meriç Deltası'nda yer almaktadır. Bu iki önemli göl, çeltik tarlalarının sulanması için de kullanılmaktadır ve tarım alanlarının drenaj suları, drenaj kanalları yoluyla tekrar bunlara deşarj edilmektedir. Ayrıca, bölgede yapılan sosyal araştırmalar, yerel halkın bu önemli su sistemleri konusunda oldukça duyarsız olduğunu göstermektedir. Bu durum deltanın her geçen gün daha da kirlenmesine ve ötrofik bir karakter sergilemesine neden olmaktadır (Tokatlı vd., 2014; Tokatlı ve Gürbüz, 2014; Tokatlı ve Baştatlı, 2016; Tokatl1, 2017; 2018; 2019).

$\mathrm{Bu}$ çalışmanın amacı, Gala ve Sığırcı göllerindeki altı balık türünün yenilebilir dokusu olan kaslarındaki EE (Li, B, Al, V, Cr, Mn, Fe, Co, Ni, Cu, Zn, As, Se, Sr, Ag, Cd, Sb, Ba, Tl ve Pb) konsantrasyonlarını belirleyerek bu balıkların yetişkinler tarafından tüketilmesi durumunda oluşabilecek potansiyel sağlık risklerini değerlendirmektir.

\section{MATERYAL VE METOT}

\section{Çalışma Alanı ve Örekelerin Toplanması}

Meriç Nehir havzasını topografik haritası ve çalışma alanı Şekil 1'de verilmiştir. Balık örnekleri [Carassius gibelio (Bloch, 1782), Carassius carassius (Linnaeus, 1758)] ve Siğırc1 [Scardinius erythrophthalmus (Linnaeus, 1758), Cyprinus carpio Linnaeus, 1758, Sander lucioperca (Linnaeus, 1758), Perca fluviatilis Linnaeus, 1758], 2016 yılının yaz mevsiminde, serpme ağlar kullanılarak temin edilmiştir. Balıklara ait bazı metrik karakterler Tablo 1'de verilmiştir. 
Tablo 1. Meriç Nehri Deltası'ndan yakalanan balıkların bazı metrik özellikleri

\begin{tabular}{cccccc}
\hline \hline \multirow{2}{*}{ Lokalite } & \multirow{2}{*}{ Balık Türü } & \multirow{2}{*}{ Ağırlık } & \multicolumn{3}{c}{ Boy $(\mathrm{mm} \pm \mathrm{SD})$} \\
\cline { 4 - 6 } & & $(\mathrm{gr} \pm \mathrm{SD})$ & Standart & Çatal & Total \\
\hline Gala & C, gibelio $(\mathrm{n}=4)$ & $552,5 \pm 9,8$ & $243,7 \pm 14,2$ & $270,2 \pm 15$ & $284,7 \pm 18,1$ \\
Lake & C, carassius $(\mathrm{n}=5)$ & $1166 \pm 105$ & $315 \pm 16,9$ & $342,2 \pm 15,6$ & $385,8 \pm 11,1$ \\
\hline \multirow{2}{*}{ Sı̆̆ı̆ırc } & S, erythroph $(\mathrm{n}=5)$ & $261,6 \pm 5,2$ & $241,4 \pm 12,1$ & $264,8 \pm 12,6$ & $287 \pm 13,5$ \\
Lake & C, carpio $(\mathrm{n}=2)$ & $1115 \pm 49,4$ & $306,5 \pm 9,1$ & $332 \pm 11,3$ & $369,5 \pm 7,7$ \\
& S, lucioperca $(\mathrm{n}=1)$ & $430 \pm 0$ & $333 \pm 0$ & $362 \pm 0$ & $375 \pm 0$ \\
& $P$, fluviatilis $(\mathrm{n}=3)$ & $189,3 \pm 14$ & $198,3 \pm 22,5$ & $216,6 \pm 16$ & $228,3 \pm 20,2$ \\
\hline \hline
\end{tabular}

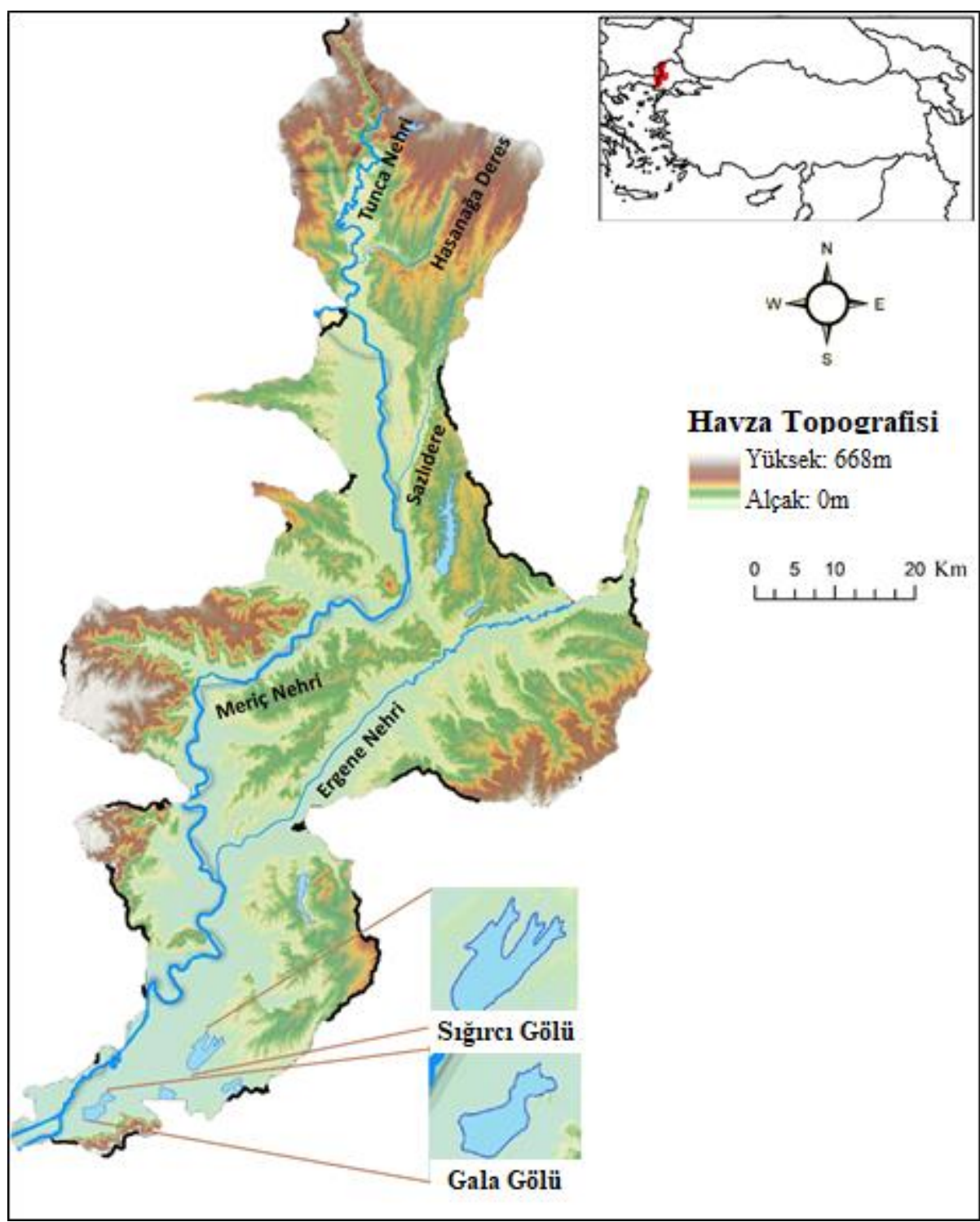

Şekil 1. Meriç Havzası ve incelenen göller

\section{Kimyasal Analizler}

Balık örneklerinin kas dokuları çıkarıldı ve her numuneden 0.50 gr alınarak CEM Mars Xpress 5 mikrodalga sindirim reaktörlerine yerleştirildi. Reaktörlere sırasıyla 1:3 oranlı $\mathrm{HCl}: \mathrm{HNO}_{3}$ asitleri eklenmiştir. Numuneler, otuz dakika boyunca $200{ }^{\circ} \mathrm{C}$ 'de mineralize edildi. Daha sonra, hacimleri 100 ml'ye ultra saf su ile tamamland. Balık dokularındaki element seviyeleri, "Agilent 7700 xx" markalı Endüktif Olarak Çiftleşmiş Plazma - Kütle Spektrometresi (ICP-MS) cihazı kullanılarak belirlenmiştir (ASTM, 1985; USEPA, 1998; 2001). 


\section{Sağlık Riski Değerlendirmesi}

Balıklarda bulunan EE'lerin yetişkin insanlardaki sağlık risklerini değerlendirmek için "Edible Daily Intake (EDI)", "Target Hazard Coefficient (THQ)", "Hazard Index (HI)" ve "Cancer Risk (CR)" hesaplanmıştır.

\section{Edible Daily Intake (EDI)}

EE'lerin yetişkinler için EDI değeri $(\mathrm{EDI}=\mathrm{mg} / \mathrm{kg}$ vücut ağılığı/gün) aşağıdaki formüle göre hesapland1 (Javed ve Usmani 2016):

$$
E D I=\frac{C_{\text {element }} \times D_{\text {food intake }}}{B W}
$$

$\mathrm{C}_{\text {element: }}$ Balık kasındaki metal konsantrasyonu ( $\left.\mathrm{mg} / \mathrm{kg}\right)$,

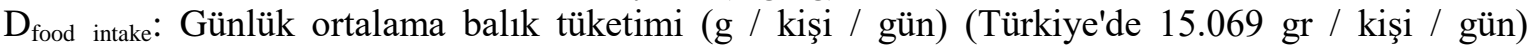
(GDFA, 2018),

BW: Ortalama vücut ağırlığıdır (yetişkinler için $70 \mathrm{~kg}$ ).

$\mathrm{Bu}$ çalışmada hesaplanan EDI değerleri, USEPA (2019) tarafindan önerilen oral referans doz $\left(\mathrm{RfD}_{\mathrm{o}}\right)$ değerleriyle karşılaş̧ırıldı.

\section{Target Hazard Coefficient (THQ)}

THQ, kirletici maruziyetine bağlı olarak risk seviyesinin (kanserojen olmayan) bir tahminidir. Her ağır metalin THQ'sunu belirlemek için kullanılan yöntem, aşağıdaki formülle hesaplanmıştır (Chien vd., 2002):

$$
T H Q=\frac{E F \times E D \times F I R \times C}{R f D_{o} \times B W \times A T} \times 10^{-3}
$$

EF: Maruz kalma sıklığı (365 gün / yıl),

ED: Maruz kalma süresi (ortalama yaşam süresi: 70 yıl),

FIR: Gıda alım oranı (20 gr / gün),

C: Balık kas dokusunda ortalama ağır metal konsantrasyonu ( $\mathrm{mg} / \mathrm{kg})$,

RfDo: Oral referans doz ( $\mathrm{mg} / \mathrm{kg} /$ day),

BW: Ortalama vücut ağırlığı (ortalama $70 \mathrm{~kg}$ ),

AT: Kanserojen olmayanlar için ortalama maruz kalma süresi (365 gün / yıl x maruziyet yılı sayıs1)

Eğer THQ değerleri 1'i aşarsa, karsinojenik olmayan olumsuz sağlık etkilerinin ortaya çıkma potansiyeli olabilir. Eğer değerler l'den düşükse, karsinojenik olmayan sağl1k etkileri beklenmez (Mehmood et al., 2020; Çulha et al., 2016).

\section{Hazard Index (HI)}

HI, gözlemlenen tüm metaller için THQ'nun toplamıdır $(\mathrm{HI}<1$ ise güvenli, $\mathrm{HI}>1$ ise tehlikeli) (Varol ve diğerleri, 2019).

$$
H I=\sum_{i=1}^{n} T H Q_{i}
$$

\section{Cancer Risk (CR)}

$\mathrm{Cr}, \mathrm{Ni}, \mathrm{Pb}$ ve As ömür boyu maruz kalma neticesinde oluşabilecek hedef kanser riski (CR) aşağıdaki denkleme göre hesaplanmıştır (Javed ve Usmani 2016):

$$
C R=\frac{E F \times E D \times F I R \times C \times C S F}{B W \times A T} \times 10^{-3}
$$

CSF: Entegre Risk Bilgi Sisteminden kanserojen eğim faktörüdür (Cr: 0,5; Ni: 1,7; Pb: 0,0085; As: $1,5 \mathrm{mg} / \mathrm{kg} / \mathrm{gün})$.

Hesaplanan CR katsayılarının değerlendirilmesi şu şekilde yapılmaktadır; $C R<10^{-6}$ : ihmal

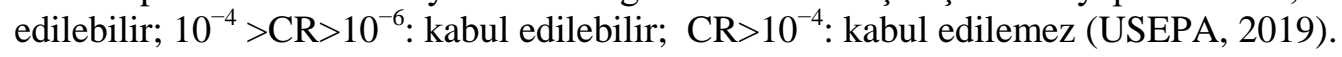




\section{SONUÇLAR VE TARTIŞMA}

Son yıllarda sanayileşme ve tarımsal faaliyetlerin artması nedeniyle Sığırcı ve Gala Göllerinin su kalitesi her geçen gün kötüleşmektedir. Bu nedenle göl sularındaki EE’ler, balıkların su ve sedimentlerle etkileşimi sonucu balık dokularında birikmektedir. Balığa dayalı bir beslenme şeklinin pek çok faydaları vardır, ancak toksik metaller, pestisit ve diğer toksinler ile kontamine olmuş balıklar tüketicilerin sağlı̆̆ını tehlikeye atabilir. Carassius gibelio, Carassius carassius, Scardinius erythrophthalmus, Cyprinus carpio, Sander lucioperca, ve Perca fluviatilis gibi türler Gala ve Sığırcı gölleri yakınlarındaki insanlar tarafından yaygın olarak tüketilmektedir. Balık türleri için hesaplanan EDI değerleri Tablo 2'de listelendi. Türler arasında tespit edilen EDI değerleri arasındaki bu farklılıklar çoğunlukla balık türlerinin yaşam ortamları, besin tercihleri, metabolizmaları ve fizyolojileri ile ilişkilidir (Roleda et al., 2019; Tepe, 2009). EDI değerleri, oral referans doz (RfD) değerleri ile karşılaştıııldı. Sonuçlar, $\mathrm{Li}, \mathrm{B}, \mathrm{Cr}, \mathrm{Co}, \mathrm{Se}, \mathrm{Tl}$ ve $\mathrm{Pb}$ için EDI değerlerinin tüm balık türlerinde genellikle izin verilen günlük dozdan daha yüksek olduğunu göstermiştir. Geri kalan metallerde (Al, V, Mn, Fe, Ni, Cu, Zn, As, Sr, Ag, Cd, Sb ve Ba) ise EDI < RfD olarak hesaplanmıştır. İnsan nüfusu aynı anda birden fazla EE'ye maruz kalmaktadır. Bu nedenle son yıllarda EE'lerin insan sağlığı üzerindeki interaktif etkileri üzerine çok sayıda çalışma yapılmıştır. Örneğin As ve $\mathrm{Pb}$ arasında sinerjik bir etkinin olduğu tespit edilmiştir ve çocuklarda $\mathrm{As}$ ve $\mathrm{Pb}$ toksisitesinin birinci hedefinin merkezi sinir sistemi olduğunu ve zihinsel engelli olma olasılığını arttırdığı bildirilmiştir (Freire vd., 2018).

Tablo 2. Tespit edilen EDI değerleri

\begin{tabular}{cccccccc}
\hline \hline & & \multicolumn{7}{c}{ EDI (mg/kg BW/gün) } \\
\hline & $\begin{array}{c}\text { RfD } \\
\text { (mg/kg/gün) }\end{array}$ & $\mathbf{1}$ & $\mathbf{2}$ & $\mathbf{3}$ & $\mathbf{4}$ & $\mathbf{5}$ & $\mathbf{6}$ \\
\hline $\mathrm{Li}$ & $2.00 \mathrm{E}-03$ & $5.18 \mathrm{E}-02$ & $4.92 \mathrm{E}-02$ & $5.18 \mathrm{E}-02$ & $5.52 \mathrm{E}-02$ & $5.30 \mathrm{E}-02$ & $5.13 \mathrm{E}-02$ \\
$\mathrm{~B}$ & $2.00 \mathrm{E}-01$ & $1.12 \mathrm{E}+00$ & $1.08 \mathrm{E}+00$ & $1.12 \mathrm{E}+00$ & $1.11 \mathrm{E}+00$ & $1.11 \mathrm{E}+00$ & $1.10 \mathrm{E}+00$ \\
$\mathrm{Al}$ & $1.00 \mathrm{E}+00$ & $4.51 \mathrm{E}-01$ & $3.70 \mathrm{E}-01$ & $3.70 \mathrm{E}-01$ & $3.26 \mathrm{E}-01$ & $3.35 \mathrm{E}-01$ & $9.31 \mathrm{E}-01$ \\
$\mathrm{~V}$ & $5.00 \mathrm{E}-03$ & $5.36 \mathrm{E}-03$ & $1.31 \mathrm{E}-03$ & $2.19 \mathrm{E}-03$ & $9.66 \mathrm{E}-04$ & $7.17 \mathrm{E}-04$ & $1.62 \mathrm{E}-03$ \\
$\mathrm{Cr}$ & $3.00 \mathrm{E}-03$ & $5.36 \mathrm{E}-03$ & $3.77 \mathrm{E}-03$ & $4.41 \mathrm{E}-03$ & $2.18 \mathrm{E}-02$ & $5.46 \mathrm{E}-03$ & $5.27 \mathrm{E}-03$ \\
$\mathrm{Mn}$ & $1.40 \mathrm{E}-01$ & $1.51 \mathrm{E}-02$ & $3.64 \mathrm{E}-02$ & $2.09 \mathrm{E}-02$ & $1.17 \mathrm{E}-02$ & $9.34 \mathrm{E}-03$ & $2.37 \mathrm{E}-02$ \\
$\mathrm{Fe}$ & $7.00 \mathrm{E}-01$ & $6.13 \mathrm{E}-01$ & $5.33 \mathrm{E}-01$ & $5.17 \mathrm{E}-01$ & $7.90 \mathrm{E}-01$ & $4.90 \mathrm{E}-01$ & $8.64 \mathrm{E}-01$ \\
$\mathrm{Co}$ & $3.00 \mathrm{E}-04$ & $5.43 \mathrm{E}-04$ & $4.91 \mathrm{E}-04$ & $4.37 \mathrm{E}-04$ & $4.37 \mathrm{E}-04$ & $3.97 \mathrm{E}-04$ & $4.17 \mathrm{E}-04$ \\
$\mathrm{Ni}$ & $2.00 \mathrm{E}-02$ & $1.01 \mathrm{E}-02$ & $1.22 \mathrm{E}-02$ & $1.28 \mathrm{E}-02$ & $7.58 \mathrm{E}-03$ & $9.37 \mathrm{E}-03$ & $6.87 \mathrm{E}-03$ \\
$\mathrm{Cu}$ & $4.00 \mathrm{E}-02$ & $1.39 \mathrm{E}-02$ & $1.19 \mathrm{E}-02$ & $1.13 \mathrm{E}-02$ & $1.99 \mathrm{E}-02$ & $1.64 \mathrm{E}-02$ & $1.08 \mathrm{E}-02$ \\
$\mathrm{Zn}$ & $3.00 \mathrm{E}-01$ & $2.68 \mathrm{E}-01$ & $1.06 \mathrm{E}-01$ & $1.35 \mathrm{E}-01$ & $1.19 \mathrm{E}-01$ & $1.83 \mathrm{E}-01$ & $1.16 \mathrm{E}-01$ \\
$\mathrm{As}$ & $3.00 \mathrm{E}-04$ & $5.12 \mathrm{E}-04$ & $2.45 \mathrm{E}-04$ & $4.23 \mathrm{E}-04$ & $3.62 \mathrm{E}-04$ & $3.16 \mathrm{E}-04$ & $2.86 \mathrm{E}-04$ \\
$\mathrm{Se}$ & $5.00 \mathrm{E}-03$ & $6.95 \mathrm{E}-03$ & $8.79 \mathrm{E}-03$ & $7.47 \mathrm{E}-03$ & $5.61 \mathrm{E}-03$ & $8.21 \mathrm{E}-03$ & $7.45 \mathrm{E}-03$ \\
$\mathrm{Sr}$ & $6.00 \mathrm{E}-01$ & $3.15 \mathrm{E}-02$ & $5.38 \mathrm{E}-02$ & $8.20 \mathrm{E}-02$ & $5.38 \mathrm{E}-03$ & $5.93 \mathrm{E}-03$ & $5.75 \mathrm{E}-03$ \\
$\mathrm{Ag}$ & $5.00 \mathrm{E}-03$ & $1.27 \mathrm{E}-02$ & $8.64 \mathrm{E}-03$ & $8.31 \mathrm{E}-03$ & $1.00 \mathrm{E}-02$ & $8.52 \mathrm{E}-03$ & $8.35 \mathrm{E}-03$ \\
$\mathrm{Cd}$ & $1.00 \mathrm{E}-03$ & $1.21 \mathrm{E}-03$ & $8.74 \mathrm{E}-04$ & $8.51 \mathrm{E}-04$ & $9.20 \mathrm{E}-04$ & $9.63 \mathrm{E}-04$ & $1.04 \mathrm{E}-03$ \\
$\mathrm{Sb}$ & $4.00 \mathrm{E}-04$ & $6.10 \mathrm{E}-03$ & $5.86 \mathrm{E}-04$ & $4.74 \mathrm{E}-04$ & $4.43 \mathrm{E}-04$ & $5.29 \mathrm{E}-04$ & $5.34 \mathrm{E}-04$ \\
$\mathrm{Ba}$ & $2.00 \mathrm{E}-01$ & $8.70 \mathrm{E}-03$ & $1.40 \mathrm{E}-02$ & $2.02 \mathrm{E}-02$ & $4.05 \mathrm{E}-03$ & $4.23 \mathrm{E}-03$ & $6.57 \mathrm{E}-03$ \\
$\mathrm{Tl}$ & $1.00 \mathrm{E}-05$ & $1.26 \mathrm{E}-03$ & $2.37 \mathrm{E}-04$ & $2.31 \mathrm{E}-04$ & $1.80 \mathrm{E}-04$ & $1.97 \mathrm{E}-04$ & $2.03 \mathrm{E}-04$ \\
$\mathrm{~Pb}$ & $3.00 \mathrm{E}-03$ & $1.46 \mathrm{E}-02$ & $1.18 \mathrm{E}-02$ & $1.22 \mathrm{E}-02$ & $1.27 \mathrm{E}-02$ & $1.51 \mathrm{E}-02$ & $1.60 \mathrm{E}-02$ \\
\hline \hline $1=C$. gibelio, 2=C. carassius, 3=S. erythrophthalmus, 4=C.carpio, 5=S. Luciope, 6=P.fluviatilis & & \\
& & & & & & &
\end{tabular}


Altı balık türünün tüketimi yoluyla EE'ler için tahmin edilen THQ değerleri Tablo 3'te sunulmuştur. Gala ve Sığırcı göllerindeki her balık türündeki EE’lerin THQ değerleri l'i geçmemiştir. Ayrıca her balık türü için metallerin toplam THQ değerleri de (HI) 1'den düşüktür. THQ ve HI sonuçlarına göre, tüm balık türlerinde bireysel veya kombine elementlerin alımından, tüketiciler için herhangi bir sağlik etkisi beklenmemektedir. Bu risk tahmini yöntemi son zamanlarda birçok araştırmacı tarafından kullanılmış, geçerli ve yararlı olduğu gösterilmiştir.

As, $\mathrm{Ni}, \mathrm{Cr}$ ve $\mathrm{Pb}$ için tahmin edilen $\mathrm{CR}$ değerleri Tablo 3 'te sunulmuştur. Tüm balık türlerinde As ve $\mathrm{Pb}$ için $\mathrm{CR}$ değerleri $10^{-6}$ dan küçük (ihmal edilebilir), $\mathrm{Cr}$ ve Ni değerleri ise $10^{-4}-10^{-6}$ (kabul edilebilir) aralığındadır. Bu nedenle Sığırcı ve Gala göllerindeki altı balık türünün tüketilmesiyle As, $\mathrm{Pb}$, Ni ve Cr'ye maruz kalma durumunda tüketiciler için kayda değer bir kanserojen riski yoktur. 
Tablo 3. THQ, HI, ve CR değerleri

\begin{tabular}{|c|c|c|c|c|c|c|c|c|c|c|c|c|}
\hline & \multicolumn{6}{|c|}{ THQ } & \multicolumn{6}{|c|}{$\mathrm{CR}$} \\
\hline & 1 & 2 & 3 & 4 & 5 & 6 & 1 & 2 & 3 & 4 & 5 & 6 \\
\hline $\mathrm{Li}$ & $2.59 \mathrm{E}-02$ & $2.46 \mathrm{E}-02$ & $2.59 \mathrm{E}-02$ & $2.76 \mathrm{E}-02$ & $2.65 \mathrm{E}-02$ & $2.56 \mathrm{E}-02$ & & & & & & \\
\hline B & $5.58 \mathrm{E}-03$ & $5.40 \mathrm{E}-03$ & $5.59 \mathrm{E}-03$ & $5.54 \mathrm{E}-03$ & $5.57 \mathrm{E}-03$ & $5.52 \mathrm{E}-03$ & & & & & & \\
\hline $\mathrm{Al}$ & 4.51E-04 & 3.70E-04 & $3.70 \mathrm{E}-04$ & $3.26 \mathrm{E}-04$ & 3.35E-04 & $9.31 \mathrm{E}-04$ & & & & & & \\
\hline $\mathrm{V}$ & $1.07 \mathrm{E}-03$ & 2.63E-04 & 4.38E-04 & $1.93 \mathrm{E}-04$ & $1.43 \mathrm{E}-04$ & $3.24 \mathrm{E}-04$ & & & & & & \\
\hline $\mathrm{Cr}$ & 1.79E-03 & $1.26 \mathrm{E}-03$ & $1.47 \mathrm{E}-03$ & 7.26E-03 & $1.82 \mathrm{E}-03$ & $1.76 \mathrm{E}-03$ & $2.68 \mathrm{E}-06$ & $1.89 \mathrm{E}-06$ & $2.21 \mathrm{E}-06$ & $1.09 \mathrm{E}-05$ & $2.73 \mathrm{E}-06$ & $2.64 \mathrm{E}-06$ \\
\hline Mn & $1.08 \mathrm{E}-04$ & 2.60E-04 & 1.49E-04 & 8.39E-05 & $6.67 \mathrm{E}-05$ & 1.69E-04 & & & & & & \\
\hline $\mathrm{Fe}$ & 8.75E-04 & 7.62E-04 & 7.39E-04 & $1.13 \mathrm{E}-03$ & 7.00E-04 & $1.23 \mathrm{E}-03$ & & & & & & \\
\hline $\mathrm{Co}$ & $1.81 \mathrm{E}-03$ & $1.64 \mathrm{E}-03$ & $1.46 \mathrm{E}-03$ & $1.46 \mathrm{E}-03$ & $1.32 \mathrm{E}-03$ & 1.39E-03 & & & & & & \\
\hline $\mathrm{Ni}$ & $5.05 \mathrm{E}-04$ & $6.11 \mathrm{E}-04$ & $6.40 \mathrm{E}-04$ & 3.79E-04 & 4.69E-04 & $3.43 \mathrm{E}-04$ & $1.72 \mathrm{E}-05$ & $2.08 \mathrm{E}-05$ & $2.18 \mathrm{E}-05$ & $1.29 \mathrm{E}-05$ & $1.59 \mathrm{E}-05$ & $1.17 \mathrm{E}-05$ \\
\hline $\mathrm{Cu}$ & $3.46 \mathrm{E}-04$ & 2.98E-04 & 2.83E-04 & 4.97E-04 & 4.11E-04 & 2.71E-04 & & & & & & \\
\hline $\mathrm{Zn}$ & 8.93E-04 & $3.54 \mathrm{E}-04$ & $4.52 \mathrm{E}-04$ & $3.95 \mathrm{E}-04$ & $6.11 \mathrm{E}-04$ & $3.86 \mathrm{E}-04$ & & & & & & \\
\hline As & $1.71 \mathrm{E}-03$ & 8.17E-04 & $1.41 \mathrm{E}-03$ & $1.21 \mathrm{E}-03$ & $1.05 \mathrm{E}-03$ & $9.54 \mathrm{E}-04$ & 7.68E-07 & $3.68 \mathrm{E}-07$ & 6.34E-07 & $5.43 \mathrm{E}-07$ & 4.74E-07 & 4.29E-07 \\
\hline $\mathrm{Se}$ & 1.39E-03 & $1.76 \mathrm{E}-03$ & $1.49 \mathrm{E}-03$ & $1.12 \mathrm{E}-03$ & $1.64 \mathrm{E}-03$ & $1.49 \mathrm{E}-03$ & & & & & & \\
\hline $\mathrm{Sr}$ & $5.25 \mathrm{E}-05$ & 8.97E-05 & $1.37 \mathrm{E}-04$ & 8.97E-06 & $9.88 \mathrm{E}-06$ & $9.58 \mathrm{E}-06$ & & & & & & \\
\hline $\mathrm{Ag}$ & $2.54 \mathrm{E}-03$ & $1.73 \mathrm{E}-03$ & $1.66 \mathrm{E}-03$ & $2.01 \mathrm{E}-03$ & $1.70 \mathrm{E}-03$ & $1.67 \mathrm{E}-03$ & & & & & & \\
\hline $\mathrm{Cd}$ & $1.21 \mathrm{E}-03$ & 8.74E-04 & $8.51 \mathrm{E}-04$ & $9.20 \mathrm{E}-04$ & $9.63 \mathrm{E}-04$ & $1.04 \mathrm{E}-03$ & & & & & & \\
\hline $\mathrm{Sb}$ & $1.53 \mathrm{E}-02$ & $1.46 \mathrm{E}-03$ & 1.19E-03 & $1.11 \mathrm{E}-03$ & $1.32 \mathrm{E}-03$ & $1.34 \mathrm{E}-03$ & & & & & & \\
\hline $\mathrm{Ba}$ & 4.35E-05 & $6.98 \mathrm{E}-05$ & $1.01 \mathrm{E}-04$ & $2.03 \mathrm{E}-05$ & $2.11 \mathrm{E}-05$ & $3.29 \mathrm{E}-05$ & & & & & & \\
\hline $\mathrm{Tl}$ & $1.26 \mathrm{E}-01$ & 2.37E-02 & 2.31E-02 & $1.80 \mathrm{E}-02$ & $1.97 \mathrm{E}-02$ & 2.03E-02 & & & & & & \\
\hline $\mathrm{Pb}$ & 4.87E-03 & $3.94 \mathrm{E}-03$ & $4.08 \mathrm{E}-03$ & $4.24 \mathrm{E}-03$ & $5.02 \mathrm{E}-03$ & $5.35 \mathrm{E}-03$ & $1.24 \mathrm{E}-07$ & $1.00 \mathrm{E}-07$ & $1.04 \mathrm{E}-07$ & $1.08 \mathrm{E}-07$ & $1.28 \mathrm{E}-07$ & $1.36 \mathrm{E}-07$ \\
\hline $\mathrm{HI}$ & $1,93 \mathrm{E}-01$ & 7,03E-02 & $7,15 \mathrm{E}-02$ & $7,35 \mathrm{E}-02$ & $6,94 \mathrm{E}-02$ & 7,01E-02 & & & & & & \\
\hline
\end{tabular}


Arsenik, balık dokusunda hem organik hem de inorganik formlarda bulunur ve inorganik As insanlar için en toksik olan formdur (Jara ve Winter, 2014). Bu nedenle As maruziyetinden kaynaklanan insan sağlığı risklerini tahmin etmek için kullanılan EDI, RfD ve CSF değerlerinin hesaplanmasında inorganik As kullanılması uygundur. Ancak bu çalışmada balık örneklerinde toplam (inorganik + organik) As ölçülmüştür. Balık tüketmenin insan sağlı̆̆ risklerini tahmin etmek için balıklarda inorganik As yüzdesi hakkında bir varsayım yapılmalıdır. Yapılan araştırmalarda çoğu balıklarda inorganik As yüzdesinin \% 0 ila \% 9,5 arasında değiştiği bildirmiştir (Varol ve Sünbül, 2020). Çalışmamızda daha koruyucu olmak için toplam arseniğin \%10'unun inorganik As olduğu varsayılmıştır.

Varol ve Sünbül (2018), mevcut çalışmamızda elde edilen veriler ile benzer şekilde, Türkiye'nin en büyük 2. baraj gölündeki beş balıkta THQ ve HI değerlerinin insan sağlığı için risk oluşturmayacak seviyede olduğunu (1 den küçük) ve iAs için kanser riski (CR) eşik değerini (10-6) aşmadığını bildirmişlerdir. Başka bir çalışmada Garnero ve ark. (2020), mevcut çalışmamızda elde edilen verilerden farklı olarak, Río Tercero Barajında (Arjantin) altı balık türündeki iAs konsantrasyonlarının izin verilen maksimum günlük konsantrasyonları aştı̆̆ını, THQ ve total THQ (HI) değerlerinin 1'in birkaç katı olduğunu, bildirmişlerdir. Ayrıca, arseniğin neden olduğu kanser riskinin kabul edilebilir değerden (10-4) fazla olduğunu, dolayısıyla bu rezervuardaki incelenen balık türlerin besin olarak tüketiminin, insanlar için toksikolojik bir riskinin olabileceğini tespit etmişlerdir.

\section{SONUÇ}

$\mathrm{Bu}$ çalışma ile Meriç - Ergene havzasının önemli sulak alanlarından Sığırcı ve Gala göllerindeki altı balık türünün (Carassius gibelio, Carassius carassius, Scardinius erythrophthalmus, Cyprinus carpio, Sander lucioperca, ve Perca fluviatilis), insanlar tarafindan besin olarak tüketilen kas dokularmdaki eser element (Li, B, Al, V, Cr, Mn, Fe, Co, Ni, Cu, Zn, As, Se, Sr, Ag, Cd, Sb, Ba, Tl ve $\mathrm{Pb}$ ) konsantrasyonları kullanılarak, balık tüketiminden kaynaklanabilecek insan sağlığı için bazı risk değerlendirmeleri yapılmıştır. Bu risk değerlendirmelerine (EDI, THQ, HI ve CR) dayanarak, TE maruz kalmanın Gala ve Sığırcı Göllerindeki balıklarla beslenen insanlar için şimdilik önemli bir sağlık riski oluşturmadığı tespit edildi. Bu çalışmanın sonuçları, balık tüketiminin olası olumsuz etkileri konusunda uyaran, ileri çalışmalar için bir başlangıç noktasıdır. Bu şekilde, bu bilgiler su ortamlarını ve onlarla etkileşime giren insanları korumak için politikaların geliştirilmesine veri sağlayabilir.

Teşekkür: Bu araştırma, Trakya Üniversitesi Bilimsel Araştırma Projeleri (TÜBAP 2016/86) tarafından mali olarak desteklenmiştir.

\section{KAYNAKLAR}

Ahmed, M. K., Baki, M. A., Islam, M. S., Kundu, G. K., Habibullah-Al-Mamun, M., Sarkar, S. K., Hossain, M. M. (2015). Human health risk assessment of heavy metals in tropical fish and shellfish collected from the river Buriganga, Bangladesh. Environmental science and pollution research, 22 (20), 15880-15890.

Alamdar, A., Eqani, S. A. M. A. S., Hanif, N., Ali, S. M., Fasola, M., Bokhari, H., Shen, H. (2017). Human exposure to trace metals and arsenic via consumption of fish from river Chenab, Pakistan and associated health risks. Chemosphere, 168, 1004-1012.

Ali, M. M., Ali, M. L., Proshad, R., Islam, S., Rahman, Z., Tusher, T. R., Al, M. A. (2019). Heavy metal concentrations in commercially valuable fishes with health hazard inference from Karnaphuli river, Bangladesh. Human and Ecological Risk Assessment: An International Journal, 1-17.

ASTM (American Society for Testing and Materials), 1985. Preparation of biological samples for inorganic chemical analysis 1, Annual Book of ASTM Standards, D-19, pp. 740- 747.

Chien, L. C., Hung, T. C., Choang, K. Y., Yeh, C. Y., Meng, P. J., Shieh, M. J., Han, B. C. (2002). Daily intake of TBT, $\mathrm{Cu}, \mathrm{Zn}, \mathrm{Cd}$ and As for fishermen in Taiwan. Science of the total environment, 285 (1-3), 177185.

Çiçek, A., Tokatlı, C., Köse, E. (2013). Ecological risk assessment of heavy metals in sediment of Felent Stream (Sakarya River Basin, Turkey). Pakisan Journal of Zoology, 45 (5): 1335-1341.

Çulha, S. T., Yabanl, M., Baki, B., Yozukmaz, A. (2016). Heavy metals in tissues of scorpionfish (Scorpaena porcus) caught from Black Sea (Turkey) and potential risks to human health. Environmental Science and Pollution Research, 23 (20), 20882-20892. 
Fang, T., Lu, W., Li, J., Zhao, X., Yang, K. (2017). Levels and risk assessment of metals in sediment and fish from Chaohu Lake, Anhui Province, China. Environmental Science and Pollution Research, 24 (18), 15390-15400.

Feng, J., Liu, R., Chen, P., Yuan, S., Zhao, D., Zhang, J., Zheng, Z. (2015). Degradation of aqueous 3, 4dichloroaniline by a novel dielectric barrier discharge plasma reactor. Environmental Science and Pollution Research, 22 (6), 4447-4459.

Freire, C., Amaya, E., Gil, F., Fernández, M. F., Murcia, M., Llop, S., Ezama, E. (2018). Prenatal co-exposure to neurotoxic metals and neurodevelopment in preschool children: The Environment and Childhood (INMA) Project. Science of the Total Environment, 621, 340-351.

Garnero, P. L., de los Angeles Bistoni, M., Monferran, M. (2020). Trace element concentrations in six fish species from freshwater lentic environments and evaluation of possible health risks according to international standards of consumption. Environmental science and pollution research international. https://doi.org/10.1007/s11356-020-08756-7

GDFA (General Directorate of Fisheries and Aquaculture) (2018). Fisheries statistics. Republic of Turkey Ministry of Food Agriculture and Livestock.

Jara, E. A., Winter, C. K. (2014). Dietary exposure to total and inorganic arsenic in the United States, 20062008. International Journal of Food Contamination, 1 (1), 3.

Jägerbrand, A. K., Sjöbergh, J. (2016). Effects of weather conditions, light conditions, and road lighting on vehicle speed. SpringerPlus, 5 (1), 505.

Kosker, A. R. (2020). Metal and fatty acid levels of some commercially important marine species from the northeastern Mediterranean: benefits and health risk estimation. Environmental Monitoring and Assessment, 192, 1-16.

Köse, E., Emiroğlu, Ö., Çiçek, A., Tokatlı, C., Başkurt, S., Aksu, S. (2018). Sediment quality assessment in Porsuk Stream Basin (Turkey) from a multi-statistical perspective. Polish Journal of Environmental Studies, 27 (2): 747-752.

Kustas, W. P., Agam, N., Alfieri, J. G., McKee, L. G., Prueger, J. H., Hipps, L. E., Heitman, J. L. (2019). Below canopy radiation divergence in a vineyard: implications on interrow surface energy balance. Irrigation Science, 37 (3), 227-237.

Li, J., Miao, X., Hao, Y., Xie, Z., Zou, S., Zhou, C. (2020). Health Risk Assessment of Metals (Cu, Pb, Zn, Cr, $\mathrm{Cd}, \mathrm{As}, \mathrm{Hg}, \mathrm{Se}$ ) in Angling Fish with Different Lengths Collected from Liuzhou, China. International Journal of Environmental Research and Public Health, 17 (7), 2192.

Maurya, P. K., Malik, D. S., Yadav, K. K., Kumar, A., Kumar, S., Kamyab, H. (2019). Bioaccumulation and potential sources of heavy metal contamination in fish species in River Ganga basin: Possible human health risks evaluation. Toxicology Reports, 6, 472-481.

Mehmood, R., Imran, U., Ullah, A., Ullman, J. L., Weidhaas, J. (2020). Health risks associated with accumulation of heavy metals in fish of Keenjhar Lake, Pakistan. Environmental Science and Pollution Research, 1-11.

Miao, X., Hao, Y., Tang, X., Xie, Z., Liu, L., Luo, S., Li, J. (2020). Analysis and health risk assessment of toxic and essential elements of the wild fish caught by anglers in Liuzhou as a large industrial city of China. Chemosphere, 243, 125337.

Paramasivam, K., Ramasamy, V., Suresh, G. (2015). Impact of sediment characteristics on the heavy metal concentration and their ecological risk level of surface sediments of Vaigai river, Tamilnadu, India. Spectrochimica Acta Part A: Molecular and Biomolecular Spectroscopy, 137, 397-407.

Roleda, M. Y., Marfaing, H., Desnica, N., Jónsdóttir, R., Skjermo, J., Rebours, C., Nitschke, U. (2019). Variations in polyphenol and heavy metal contents of wild-harvested and cultivated seaweed bulk biomass: Health risk assessment and implication for food applications. Food Control, 95, 121-134.

Simpson, S. L., Batley, G. E., Chariton, A. A., Stauber, J. L., King, C. K., Chapman, J. C., Maher, W. A. (2005). Handbook for sediment quality assessment. Bangor, NSW: Centre for Environmental Contaminants Research.

Tepe, Y. (2009). Metal concentrations in eight fish species from Aegean and Mediterranean Seas. Environmental monitoring and assessment, 159, 501.

Tokatl, C. (2015). Assessment of the water quality in the Meriç River: as an element of the ecosystem in the Thrace Region of Turkey. Polish Journal of Environmental Studies, 24 (5): 2205-2211.

Tokatlı, C. (2017). Bio - Ecological and Statistical Risk Assessment of Toxic Metals in Sediments of a Worldwide Important Wetland: Gala Lake National Park (Turkey). Archives of Environmental Protection, 43 (1): 34-47.

Tokatlı, C., Baştatlı, Y. (2016). Trace and Toxic Element Levels in River Sediments. Polish Journal of Environmental Studies, 25 (4): 1715-1720.

Tokatl1, C. (2019). Sediment Quality of Ergene River Basin: Bio - Ecological Risk Assessment of Toxic Metals. Environmental Monitoring and Assessment, 191 (11): 1-12. 
Tokatl1, C. (2018). Essential and Toxic Element Bioaccumulations in Fishes of Gala and Siğirci Lakes (Meriç River Delta, Turkey). Acta Alimentaria, 47 (4): 470-478.

Tokatl, C., Gürbüz, E. (2014). Socioeconomical and Socioecological Assessment on the Perceptions of Local People Of The Enez and Yeni Karpuzlu Districts (Edirne) on the Gala Lake National Park. International Journal of Social and Economic Sciences, 4 (2): 01-05.

Tokatlı, C., Köse, E., Uğurluoğlu, A., Çiçek, A., Emiroğlu, Ö. (2014). Gala Gölü (Edirne) Su Kalitesinin Coğrafi Bilgi Sistemi (CBS) Kullanilarak Değerlendirilmesi. Sigma Journal of Engineering and Natural Sciences, 32: 490-501.

Türkmen, M.,Tepe, Y., Türkmen, A., Ateş, A., 2012. Investigation of Metals in Tissues of Fish Species from Akyatan Lagoon. Fresenius Environmental Bulletin, 21, (11c): 3562-3567.

Türkmen, M., Türkmen, A., Tepe, Y., Ateş, A., Gökkuş, K., 2008. Determination of Metal Contaminations in Sea Foods from Marmara, Aegean and Mediterranean Seas: Twelve Fish Species. Food Chemistry, 108: 794-800.

Türkmen, A., Türkmen, M.,Tepe, Y., Çekiç, M., 2010. Metals in Tissues of Fish from Yelkoma Lagoon, Northeastern Mediterranean. Environmental Monitoring and Assessment, 168 (1-4), 223-230.

USEPA (U.S.Environmental Protection Agency EPA) (1998). METHOD 3051, Microwave Assisted Acid Digestion of Sediments, Sludges, Soils, and Oils.

USEPA (U.S.Environmental Protection Agency) (2001). METHOD 200.7, Determination Of Metals And Trace Elements In Water And Wastes by Inductively Coupled Plasma-Atomic Emission Spectrometry.

USEPA (U.S. Environmental Protection Agency) (2019). Regional Screening Levels (RSLs) -Equations. USEPA. https://www.epa.gov/risk/regional-screening-levels-rsls-equations, Accessed date: 20 January 2020.

Ustaoğlu, F., Islam, M. S. (2020). Potential toxic elements in sediment of some rivers at Giresun, Northeast Turkey: A preliminary assessment for ecotoxicological status and health risk. Ecological Indicators, 113, 106237.

Ustaoğlu, F., Tepe, Y., Aydin, H. (2020). Heavy metals in sediments of two nearby streams from Southeastern Black Sea coast: Contamination and ecological risk assessment. Environmental Forensics, 21 (2), 145156.

Ustaoğlu, F., Tepe, Y., Taş, B. (2019). Assessment of stream quality and health risk in a subtropical Turkey river system: A combined approach using statistical analysis and water quality index. Ecological Indicators, $113,105815$.

Varol, M., Kaçar, E., \& Akın, H. K. (2020). Accumulation of trace elements in muscle, gill and liver of fish species (Capoeta umbla and Luciobarbus mystaceus) in the Tigris River (Turkey), and health risk assessment. Environmental Research, 109570.

Varol, M., Kaya, G. K., \& Sünbül, M. R. (2019). Evaluation of health risks from exposure to arsenic and heavy metals through consumption of ten fish species. Environmental Science and Pollution Research, 26 (32), 33311-33320.

Varol, M., Sünbül, M. R. (2020). Macroelements and toxic trace elements in muscle and liver of fish species from the largest three reservoirs in Turkey and human risk assessment based on the worst-case scenarios. Environmental Research, 109298.

Varol, M., \& Sünbül, M. R. (2018). Multiple approaches to assess human health risks from carcinogenic and non-carcinogenic metals via consumption of five fish species from a large reservoir in Turkey. Science of the Total Environment, 633, 684-694.

Yin, J., Wang, L., Liu, Q., Li, S., Li, J., \& Zhang, X. (2020). Metal concentrations in fish from nine lakes of Anhui Province and the health risk assessment. Environmental Science and Pollution Research, 27, 20117-20124

Zerizghi, T., Yang, Y., Wang, W., Zhou, Y., Zhang, J., Yi, Y. (2020). Ecological risk assessment of heavy metal concentrations in sediment and fish of a shallow lake: a case study of Baiyangdian Lake, North China. Environmental Monitoring and Assessment, 192 (2),154. 